\title{
Treatment of Organic Compounds Resulting from Oil Refineries under Solar Light and Reuse it for Industrial Purpose
}

\author{
Shyamaa A. Mohammed ${ }^{\mathrm{a}, *}$, Sundus S. Al-Azawiey ${ }^{\mathrm{b}}$ and Ahmed Hasan Ali \\ ${ }^{a}$ Chemistry Department, College of Science, Al Muthanna University, Samawa, Iraq \\ ${ }^{b}$ Electromechanical Department, University of Technology, Baghdad, Iraq \\ ${ }^{c}$ College of Engineering, Al-MuthannaUniversity, Samawa, Iraq \\ *Corresponding author E-mail: shiemaa@mu.edu.iq
}

\begin{abstract}
This paper was aimed to examine the removal of oil from water by photocatalytic advance oxidation under solar light. A $\mathrm{pH}$ of 11 has shown a higher oil removal efficiency. Oil remove efficiency levels at $\mathrm{pH} 11$ have been found 75\% within 230 minutes' oily water effluent, studies have shown that Langmuir isotherm has been the best-fit one of the isotherms for the removal of oil. Data have shown a higher adsorptive capacity. Also we have studied another parameter like (catalyst concentration, initial oil concentration and $\mathrm{pH}$ ). While the treatment of waste-water is dependent upon a great level of quality for the processed industrial water, the nature of wastes that are added throughout the utilization, and the treatment degree that has been received by the waste-water. Then, the data of the waste-water quality that has been measured routinely and it was reported at the plants of the waste-water treatments are mainly for disposal of the treated effluent or the necessities of the discharge in terms of the gross pollution parameters.
\end{abstract}

Keywords: Photocatalytic Treatment; Refinery; Wastewater Treatment; ZnO photocatalyst.

\section{Introduction}

Waste-water from the petroleum refineries has a described high concentration level of the aliphatic and the aromatic petroleum hydro-carbons that result in the heavy pollutions on rivers and soil surfaces. The conventional refinery waste-water treatments have been based upon mechanical, physicochemical approaches, and additional biological treatments in treatment unit of the integrated activated sludge. Numerous solutions have been suggested in this matter, which included using the coagulants $[1,2]$.

The oils found in the polluted water may be lubricants, fats, cutting liquids, heavy hydro-carbons like the grease, tars, diesel oil, crude oils, and the light hydro-carbons like the jet fuel, kerosene, and gasoline. The main industrial oily waste sources include the metal manufacture and machining, petroleum refineries, and food processors. The machine plants and manufacturing usually use the machining coolants in the metal cutting, rolling, forming, and finishing processes. Those coolants are usually made up of the chemically stabilized oil-in-water emulsions with a 2\%-5\% oil concentration [3-5].

The present study described a photocatalytic degradation approach for treating hydro carbonic compounds in aqueous solution. The properties of this approach, which include the critical factors have been discussed. Petrol waste-water has been employed to reuse in industrial purposes in same refinery.

\section{Materials and methods}

\subsection{Materials}

In present study oily wastewater effluent was collected from Samawah refinery. Commercial ZnO powder were gained from Merck Co. (Germany Company) and has been applied without additional purifications. Distilled water was deployed for preparing different solution types. $\mathrm{HCl}(0.1 \mathrm{~N})$ and $\mathrm{NaOH}(0.1 \mathrm{~N})$ have been used for change $\mathrm{pH}$ of solution. 


\subsection{Instruments}

Photo-chemical degradations have been accomplished in specially designed insulation walled reaction containers with volume $1000 \mathrm{ml}$ under Solar-light, so the spectra were with UVvis spectro-photometer (Shimadzu UV2101 PC).

\subsection{Irradiation experiments}

In beaker $250 \mathrm{ml}$ of the oily solution $(80 \mathrm{ppm})$, photocatalyst was added with different concentration and a suspension was an exhibit to irradiation. While experiments were carried out under sunlight. Aqueous suspension has been magnetically stirred throughout experiment. At a variety of the time intervals, an aliquot had been ejected utilizing a syringe and filtered then via a Millipore syringe filter of $0.450 \mu \mathrm{m}(6,7)$.

\section{Results and discussions}

\subsection{Effect of photocatalyst concentrations}

The catalyst concentration effected on degradation of oily wastewater (80ppm) was precise utilize commercial zinc oxide between $(1 \mathrm{~g} / \mathrm{l}$ and $5 \mathrm{gll})$ maintaining the rest of the parameters like temperatures, $\mathrm{pH}$, and concentrations of the oil constant. It can be seen from the results in Figure1 that there has been an increase in the degradation rate with the increase in commercial zinc oxide concentration up to $3 \mathrm{gll}$. which can be noted in term of the availability in the active catalyst surface sites and the sunlight permeation into suspension. There has been an increase in the total active surface area with increased catalyst dosage. Altogether, due to an increased level of suspension turbidity, there has been a decreased permeation of the solar-light because of the increased effects of the scattering and thus volume of the photoactivated suspension has been reduced. Also, due to the high catalyst load, it was difficult to maintain the homogeneity of the suspension that results from particles' agglomeration, decreasing the amount of the active sites $(8,9)$.

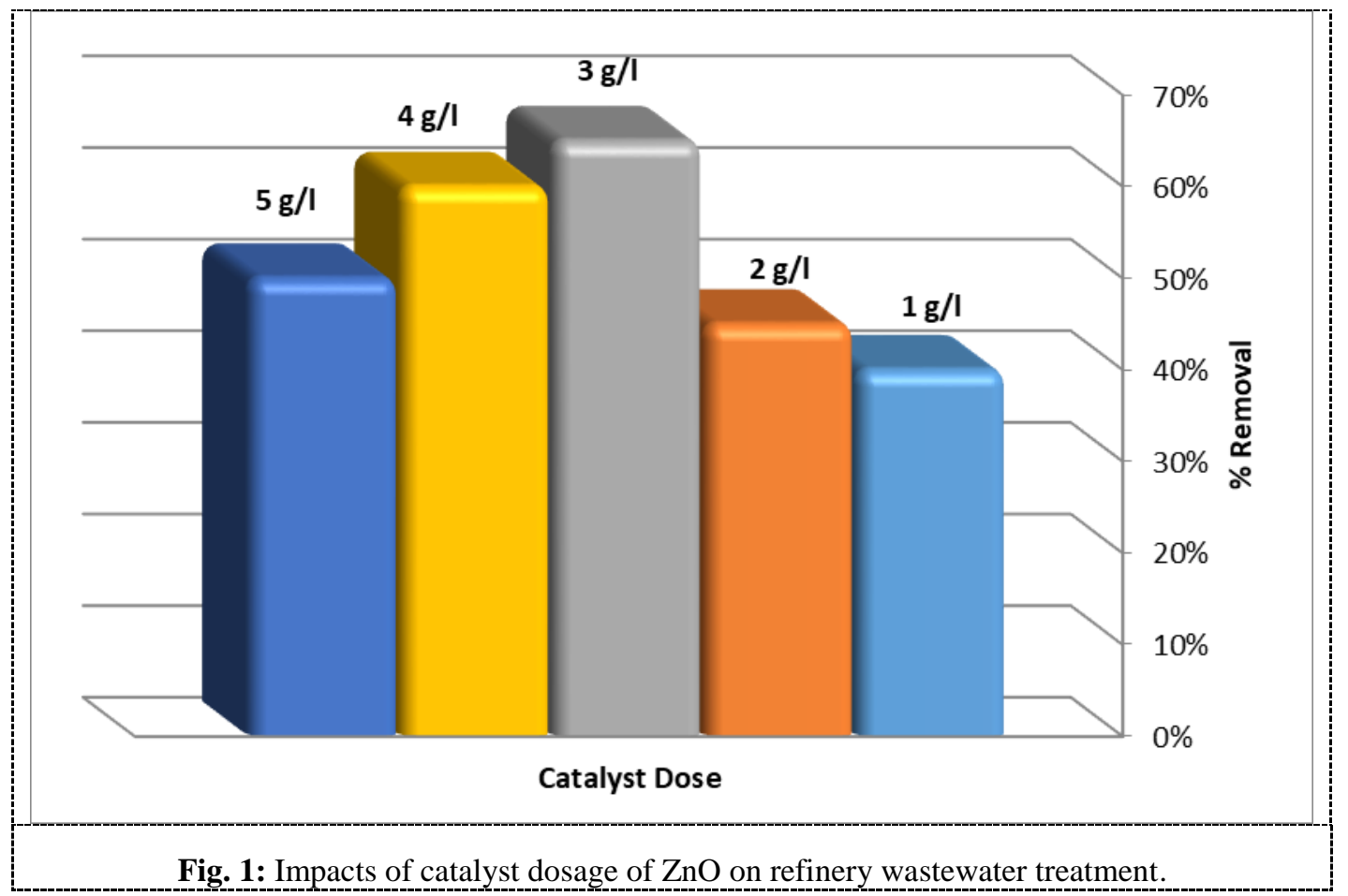

\subsection{PH effect of solution treatment}

The waste-water is created at different $\mathrm{pH}$ values, thus, examining $\mathrm{pH}$ has been found more advantageous on the photodegradation of oily wastewater. So tests were carried out at various $\mathrm{pH}$ values, approximately 3-12 for $80 \mathrm{mg} / \mathrm{L}$ oily solution concentration and for best of catalyst con. $3 \mathrm{~g} / \mathrm{l}$ of $\mathrm{ZnO}$. But the figure 2 shows the percentage treatment of against values of $\mathrm{pH}$. Exhibited increase in $\mathrm{pH}$ up to 10 for $\mathrm{ZnO}$ because of the increase of the photo-degradation activities (10). Whereas the use of zinc oxide as a catalyst has been found very proper at high $\mathrm{pH}$ values with the refinery effluent. The relation of the effects of the $\mathrm{pH}$ on the decolonization efficiency is a highly complicated subjects, because more reactions might occur to oily degradations like the "direct oxidation by positive hole, hydroxyl radical reaction, and direct reduction by electron in conduction band". Therefore, the significance of every one of them is dependent on the nature of the substrate and the values of the $\mathrm{pH}(11,12)$. 


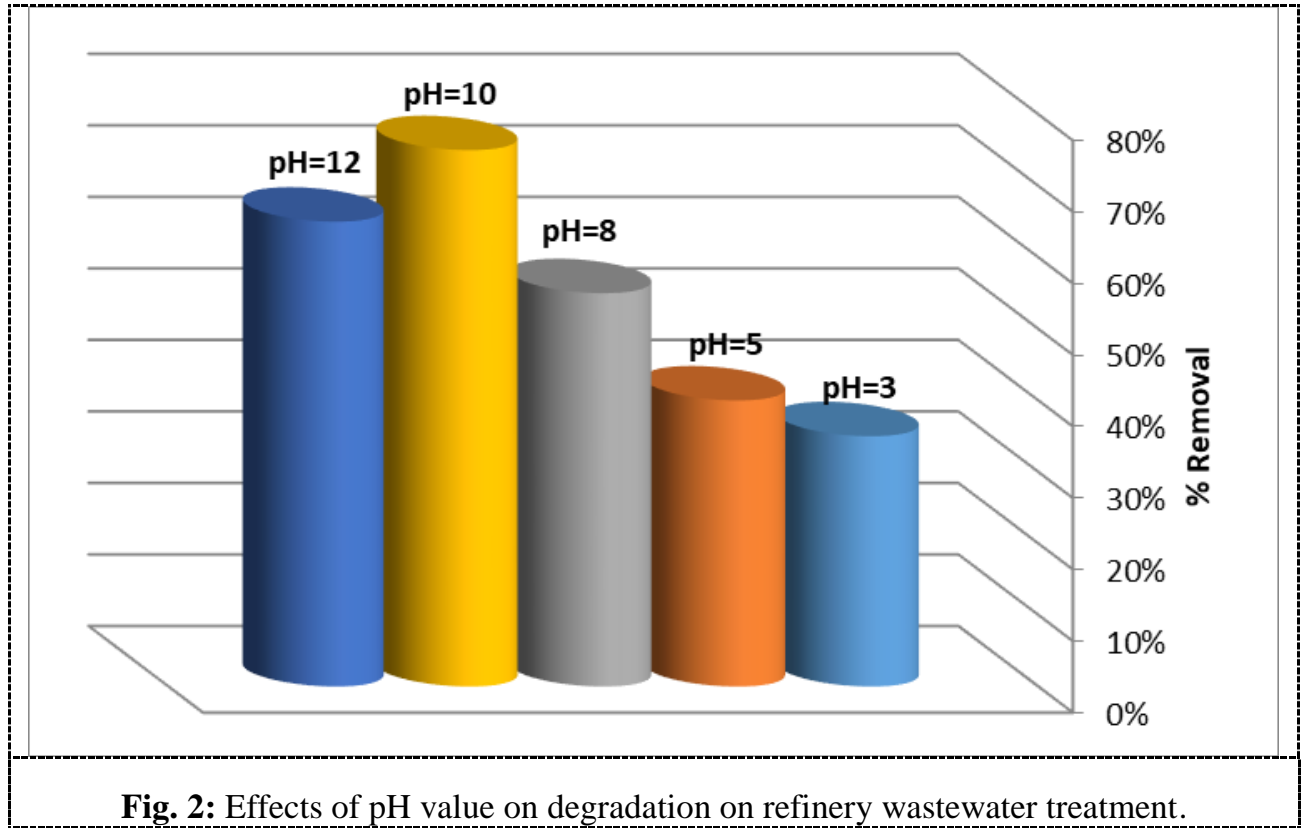

\subsection{Initial oil concentration effect}

The photodegradation of oily wastewater has been performed through the variation of initial concentration of oil from $40 \mathrm{mg} / \mathrm{l}$ so as to determine effected of initial oil concentration in wastewater on the optimal kind of the catalyst and zinc oxide dose. Increasing oil concentration has been while the percentage of degradation has been decreased refer either to increased catalyst concentration or time span for complete removal. The figure 3 depicts time-dependent graphs of degradation of oil at a variety of the concentration levels of oily solutions $40-120 \mathrm{mg} / \mathrm{l}$. none-the-less, in the case of $40 \mathrm{mg} / \mathrm{L}$ and $60 \mathrm{mg} / \mathrm{L}$, oil solutions 90 and $85 \%$ degradation had happened within $100 \mathrm{~min}$. and 150 minutes respectively. While in case of 80,100 and $120 \mathrm{mg} / \mathrm{L}$, a degradation with $230 \mathrm{~min}$. are 75, 60 and 55\%, for a complete degradation of oily water and the rate degradation had been additionally decreased with increasing the oil concentrations. Ultimately, the reasons for this behaviour have been the path length of photons entering solution decreases in high oil concentrations, and that means photochemical reactions are decreased, none-the-less, the numbers of photons that were absorbed by catalyst has been found large at low initial concentration of oil, the number of the photons, which were absorbed by catalyst have been found smaller $(13,14)$.

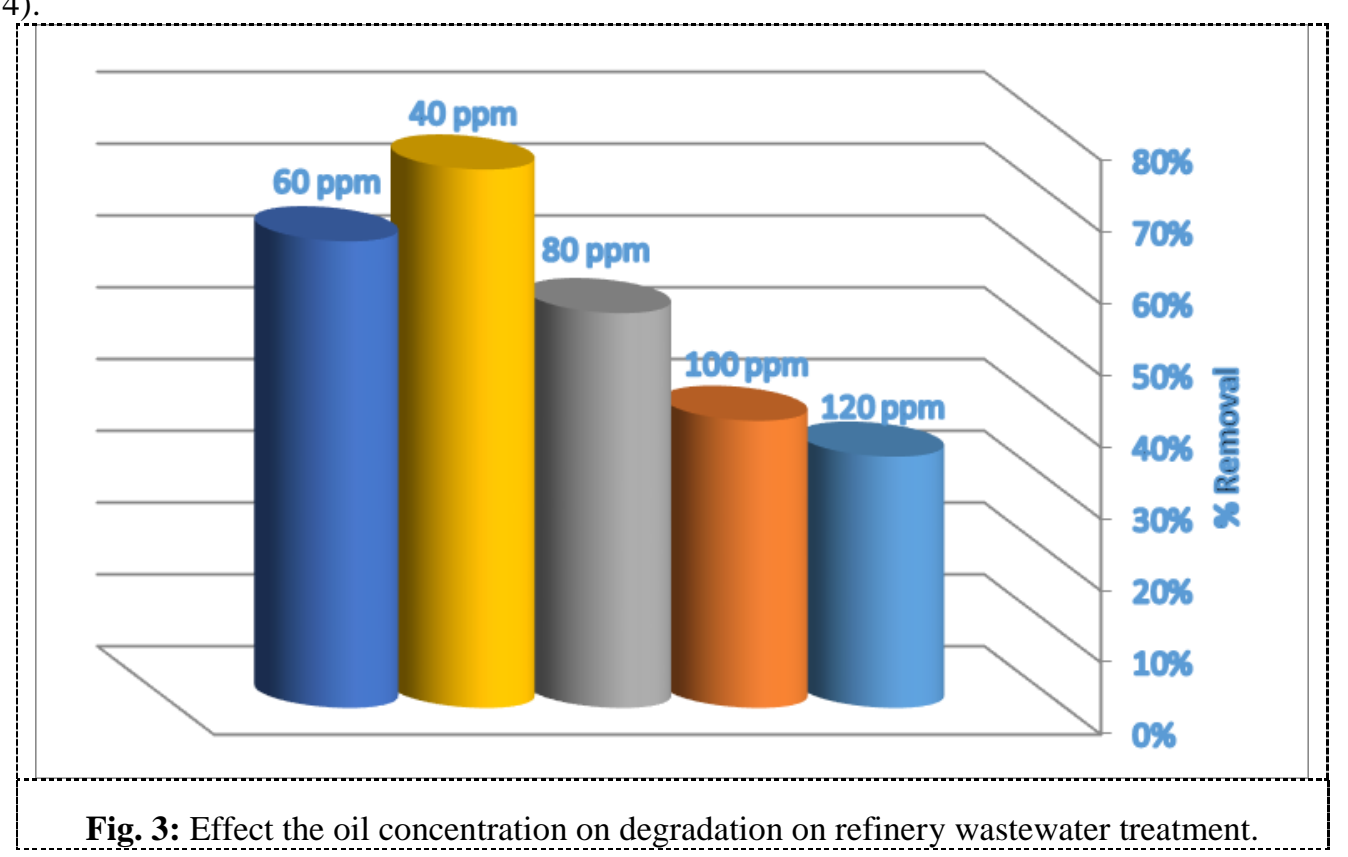




\subsection{Study of kinetic}

Figure 4 shows the kinetic study evanescence of oil concentration for an initial $80 \mathrm{mg} / \mathrm{l}$ concentration. The results have explained that the photocatalytic degradation of oily wastewater with commercial powder $\mathrm{ZnO}$ concentration $3 \mathrm{~g} / \mathrm{l}$ may be characterized by 1 st order kinetic model, in $(\mathrm{C} 0 / \mathrm{C})=\mathrm{kt}$, where $\mathrm{C}$ represents the concentration at any time $\mathrm{t}(\mathrm{y}=0.0273 \mathrm{x}-$ 0.660) and $\mathrm{C} 0$ represents the initial concentration. However, semi-logarithmic plots of concentration data have given a straight line. After that, the constant of the correlation for fitted line has been estimated as R2 $=0.8023$ for commercial $\mathrm{ZnO}(15,16)$.

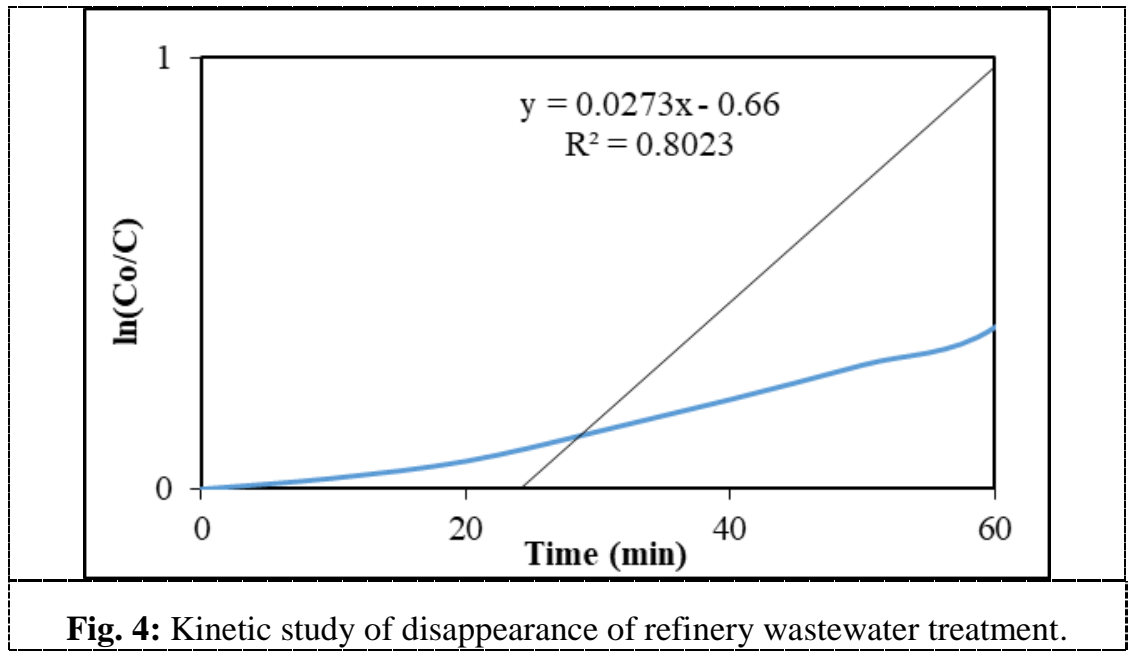

\subsection{Characteristics of the treated waste-water}

The guidelines of the waste-water reuse that have been enacted in 1918 in California might have been the first ones of this kind. Comparison of real with waste-water treated characteristics. results explain the treated waste-water characteristics near the real industrial waters, meaning that wastewater that had been treated can be re-used for industrial applications [1820], which can been seen from table1.

Table 1: Guide-lines for Water Quality Interpretations for Industrial wastewater treatments

\begin{tabular}{|c|c|c|}
\hline \multirow{2}{*}{ Properties } & Real Water & Treated Waters \\
\hline & Severe & After the Treatment \\
\hline & $>3.0$ & 2 \\
\hline \multicolumn{3}{|l|}{$\mathrm{EC}_{\mathrm{w}}(\mathrm{ds} / \mathrm{m})$} \\
\hline TDS (mg/l) & $>2,000$ & 1420 \\
\hline & $>30$ & 18 \\
\hline \multicolumn{3}{|l|}{ Nitrogen $\left(\mathrm{NO}_{3}-\mathrm{N}\right)(\mathrm{mg} / \mathrm{l})$} \\
\hline \multicolumn{3}{|l|}{ Bicarbonate $\left(\mathrm{HCO}_{3}\right)(\mathrm{me} / \mathrm{l})$} \\
\hline & $>8.5$ & 7 \\
\hline \multicolumn{3}{|l|}{ (overhead sprinkling only) } \\
\hline
\end{tabular}

\section{Conclusion}

The photo-catalytic degradations of the aqueous solutions of oily water have been observed with the use of the solar-lightirradiated catalyst of the zinc oxide. It has been found that this process yields decolorization and, then to full mineralizations of the oil solution. Also, the evolution of intermediate product and the final on photo-catalyst's surface and solution has been noticed with numerous methods, which has enabled identifying path-way of reaction, from oil the adsorptions of molecules on the surface of photo-catalyst, to ultimate products' formation. Finally, the photocatalytic degradation followed pseudo-first order kinetics. 


\section{References}

[1] (1) M. Rebhun, R. Kalabo, L. Grossman, J. Manka, C. Rav-Acha, Sorption of organics on clay and synthetic humic-clay complexes simulating aquifer processes,Water Res. 26 (1992) 79-84.

[2] (2) S. Demirci, B.R. Erdogan, Wastewater treatment at the petroleum refinery, Kirikkale, Turkey using some coagulants and Turkish clays as coagulant aids, Water Res. 32 (1998) 3495-3499.

[3] (3) B. Tensel, J. Regula, Coagulation enhanced centrifugation for treatment of petroleum hydrocarbon contaminated waters, J. Environ. Sci. Health A 35 (2000) 1557-1575.

[4] (4) S. Elmaleh, N. Ghaffor, Upgrading oil refinery effluents by cross flowultrafiltration,

[5] Water Sci. Technol. 34 (1996) 231-238.

[6] (5) T. Leiknes, M.J. Semmens, Membrane filtration for preferential removal of mulsified oil from water, Sci. Technol. 41 (2000) $101-108$.

[7] (6) Ahmed H. A., Naser G. F. and Alkaim A. F. (2018) Treatment of Textile Industries Wastewater by Advance Oxidation Process. J. Eng Appl. Sci.; (13) (1042-1045).

[8] (7) Ahmed H. A. (2017). Preparation and Characterization of Nanoparticles TiO2 and Its Application for Methylene Blue Oil Degradation under Solar Light. J. of Glob. Pharma Techno. ; (9) (141-148)

[9] (8) Ahmed H. A, Naser G. F. and Mohammed S. A. (2016). Photocatalytic Degradation of Methyl Orange Oil using Different Photocatalysts under Solar Light. Internat. J. Chem Tech Res.; (9) (10-15).

[10] (9) Hussein A. and Scholz M. (2017). Oil wastewater treatment by vertical-flow constructed wetlands. Ecol. Eng.; (101) (28-38).

[11] (10) Hussein A. and Scholz M. (2017). Treatment of artificial wastewater containing two azo textile oils by vertical-flow constructed wetlands. Environ. Sci. and Poll. Res.; (1) (1-20).

[12] (11) Ahmed H. A., Seema K., Sushil K. K. (2011) Studies on the photo catalytic decolonization of pararosanilne chloride oil and its simulated oil bath effluent. Desalination and Water Treatment. ; (25) (268-275).

[13] (12) Sushil K. K., Ahmed H. A., Seema K., Detlef W. B. (2011). Synthesis of flower like zinc oxide nanostructure and its application as a photo catalyst. Separation and Purification Technology; (80) (125-130).

[14] (13) Sushil K. K., Ahmed H. A., Seema K., Detlef W. B. (2013). Preparation, Characterization and Photo catalytic Activity of Nano sized ZnO for the Degradation of Rhoda mine B Oil and Simulated Oil bath Effluent. Science of Advanced Materials. (5) (1-7).

[15] (14) Ahmed S., Alaa M.; Nada M. A., Osman T. A., Khattab A. (2018). Photocatalytic degradation of organic oils using composite nanofibers under UV irradiation. Applied Nanoscience; (8) (155-161).

[16] (15) Zhu C., Wang L., Kong L., Yang X., Wang L., Zheng S., Chen F., Maizhi F., Zong H. (2000). Photocatalytic degradation of azo oils by supported TiO2 and UV in aqueous solution. Chemosphere; (41) (303-309).

[17] (16) Kansal S.S, Ahmed H. A., Seema K. (2010). Photocatalytic decolorization of biebrich scarlet oil in aqueous phase using different nanophotocatalysts. Desalination; (259) (147-155). 